\title{
INTER-METACARPAL BONE GRAFT FOR THENAR PARALYSIS
}

\author{
Technique and End-results
}

\author{
Donal M. Brooks, London, England \\ From the Institute of Orthopaedics, Royal National Orthopaedic Hospital
}

In 1930 Foerster described an operation for restoring opposition of the thumb in cases of paralysis of abductor brevis and opponens pollicis; a bone graft was inserted between the first two metacarpals to maintain the thumb in palmar abduction and opposition. The method was elaborated by Thompson (1942) and the results in seven cases were reviewed. Both authors were satisfied that the function of the hand was considerably improved. The alternative operation of fusing the trapezio-metacarpal joint was tried by Stiles and ForresterBrown (1922), but they found, as did Lyle (1926), that the grip between the thumb and index was weak. That this is probably due to increased movement at the trapezio-carpal joint is substantiated by Müller (1949) who has found that a good range of movement of the thumb is retained after arthrodesis of the trapezio-metacarpal joint for osteoarthritis.

This paper is based on a review of sixteen cases in which bone graft operations were performed for thenar paralysis from peripheral nerve lesions. Some unexpected sequelae have been encountered and have led to certain modifications in technique.

Indications for operation-The sole indication for an opponens bone graft is permanent paralysis of the abductor brevis and opponens pollicis muscles for which tendon transplantation to restore active opposition cannot be performed. When flexor pollicis brevis and abductor pollicis longus are working strongly, control of the thumb is often good and no reconstructive operation is necessary. As Highet (1943) and Rowntree (1949) have shown, flexor brevis is often supplied by the ulnar nerve and may therefore be spared even in complete median palsy.

The employment of the tendon of flexor sublimis to the ring finger as a motor tendon for opposition of the thumb has become almost as reliable as tendon transplantation for radial palsy; flexor or extensor carpi ulnaris, though less satisfactory, can also be used (Bunnell 1948, Irwin 1942, Thompson 1922). A tendon for transplantation is not available when these three muscles are either paralysed or weak. Lack of independent movement of muscles that have recovered after suture of a main nerve is a well-known phenomenon. Thus, after high suture of the median nerve, even though flexor profundus and flexor sublimis digitorum may contract strongly together, there is loss of independent contraction of flexor sublimis-one of the special merits of this muscle. Furthermore, scarring about the wrist may make tendon transplantation impossible. Under such circumstances stabilisation of the first metacarpal bone in the best position is indicated.

Just as in tendon transplantation, any fixed adduction contracture, particularly of the skin, should be overcome before bone-grafting. If the deformity is secondary to shortening in the adductor pollicis muscle alone, it may be corrected either at operation or by means of preliminary stripping of the first metacarpal. The preliminary stripping was done in cases where there seemed to be some prospect of recovery in abductor pollicis brevis; in a few cases power returned soon after the over-stretching of the abductor had been corrected and there was then no need to proceed to the grafting operation. If the patient is in doubt about the benefit he will derive from the operation, trial plaster fixation of the thumb in the position of function will help him to make up his mind.

\section{THE OPERATION}

A graft from the subcutaneous border of the ulna is inlaid as a strut between the first and second metacarpals so as to control abduction and rotation of the first metacarpal with the thumb in the optimal position. A tibial graft was used on six occasions, but later the 
ulna was found to be a better donor site and provided a graft of convenient size and shape which was used in a further nine cases. A segment of rib was satisfactorily employed once. Technique-Two curved incisions are made over the first and second metacarpals so that the scars do not overlie the graft at any point (Fig. 1A). The shafts of the metacarpals are exposed subperiosteally, and if there is any contracture of the adductor pollicis the muscle is divided at its insertion and full passive correction obtained. The terminal part of the radial artery is not exposed deliberately nor indeed should it be seen. A narrow osteotome is passed across the space deep to the first dorsal interosseous muscle so that it lies over the middle of the shaft of each bone; the thumb is then placed in full palmar abduction and the length of graft required is noted.

The ulna is exposed subperiosteally through a three-inch vertical incision over the lower third of the subcutaneous border and a graft of the required length is cut with a motor saw.

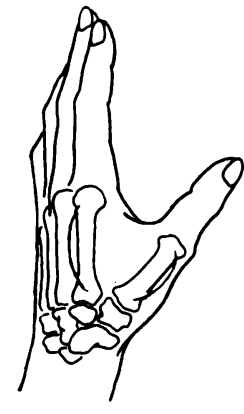

A

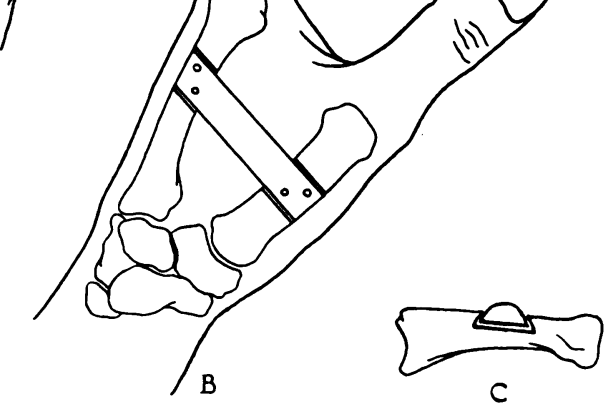

FIG. 1

Details of operation, showing the curved incisions(A), the graft in position with drill-holes ready for sutures (B) and the cross-section of graft and undercut edges of the bed $(\mathrm{C})$.

This gives a piece of bone that is D-shaped in cross-section and rather more than a centimetre in width. The incision is then closed.

The graft is passed across the interspace and, with the thumb in full abduction and rotation, the lines of bone resection are marked on the metacarpals, with particular attention to obtaining maximal rotation of the first. Saw cuts are then made so that the edges of each bed are slightly undercut (Fig. 1C); steps about half the diameter of each bone are removed. It is important to ensure that the beds for the graft are in the same plane when the thumb is in full opposition.

The graft is then driven in from one end; because of the shape of its crosssection it is held snugly in the slots and rotation is controlled at once. Two drill-holes are made through the graft and metacarpal at each end and chromic catgut sutures are inserted to maintain full abduction (Fig. 1B). The ends of the graft are trimmed to leave no rough surfaces. When subluxation of the trapezio-metacarpal joint is present, as may occur in young patients after poliomyelitis, the joint may be excised.

Closure is effected by skin sutures only, and a plaster-of-Paris cast is applied. Three weeks later the stitches are removed and a closely fitting plaster is applied for a further period of ten weeks, making three months in all.

\section{DISGUSSION OF RESULTS}

The operation has been performed in sixteen cases. In five the result was an unqualified success (Figs. 2 and 3 ); in a further nine, despite some imperfections, the function of the hand was considerably improved. The other two cases were failures.

Causes of failure and poor results-These were: 1) failure of fusion; 2) sepsis; 3) failure to achieve full opposition; 4) combined paralysis of the first dorsal interosseous and lumbrical muscles; and 5) " pollex valgus." 


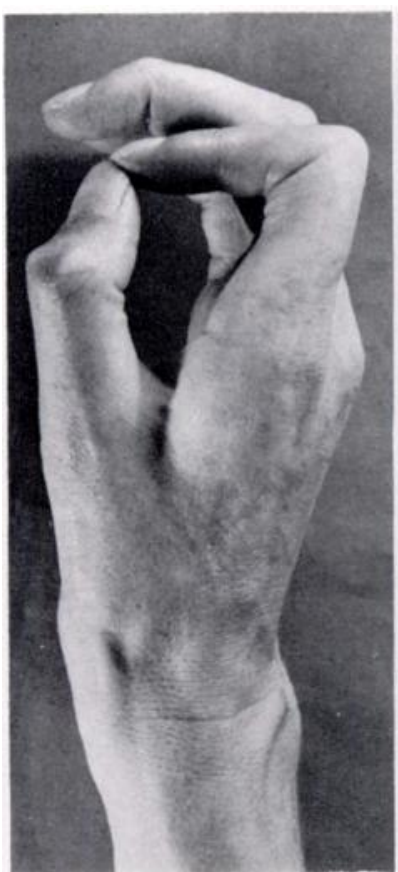

A

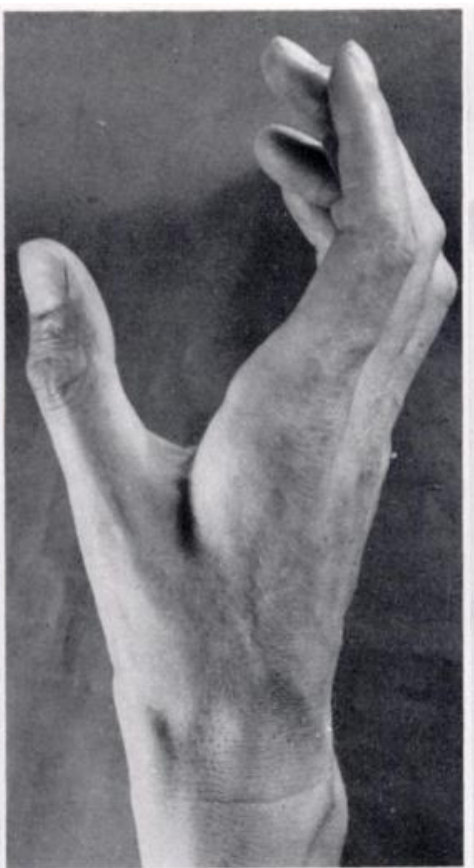

I3

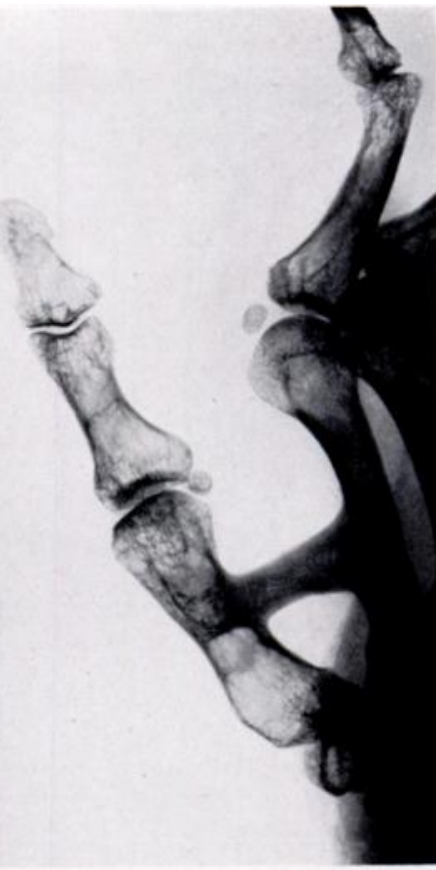

(

Fis. 2

Case 196. Note the good abduction and rotation of the thumb and the normal opposition of the terminal phalanges.

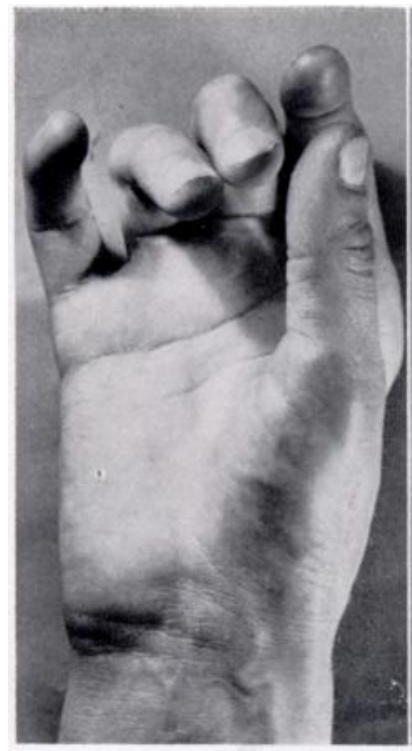

$A$

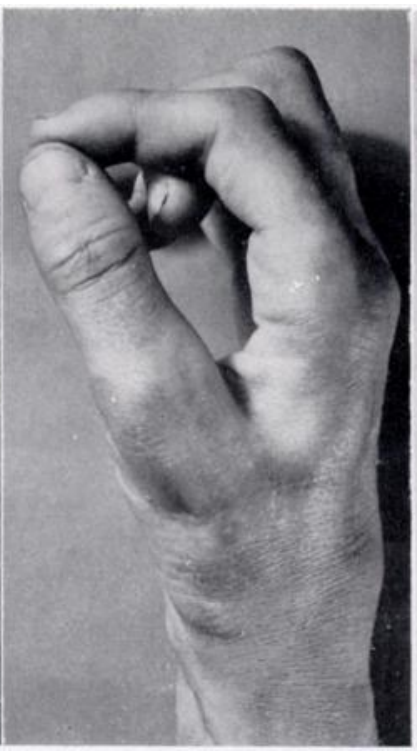

13

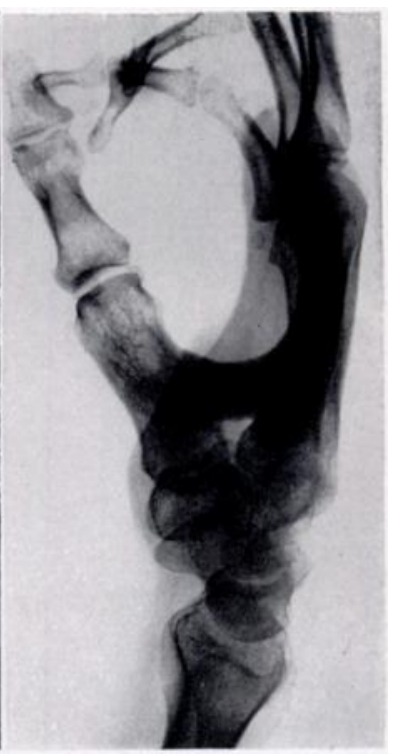

c

Fiti. 3

Case (i34. Showing a good result despite partial sequestration of the graft.

vol. 31 B, so, 4, NoYkMB!R 1949 


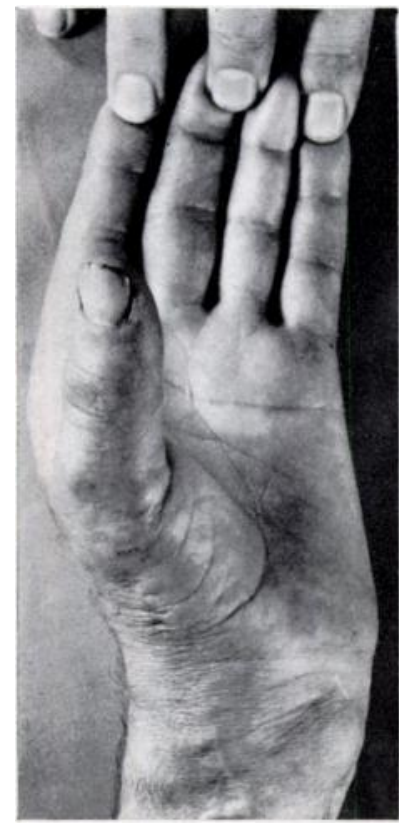

A

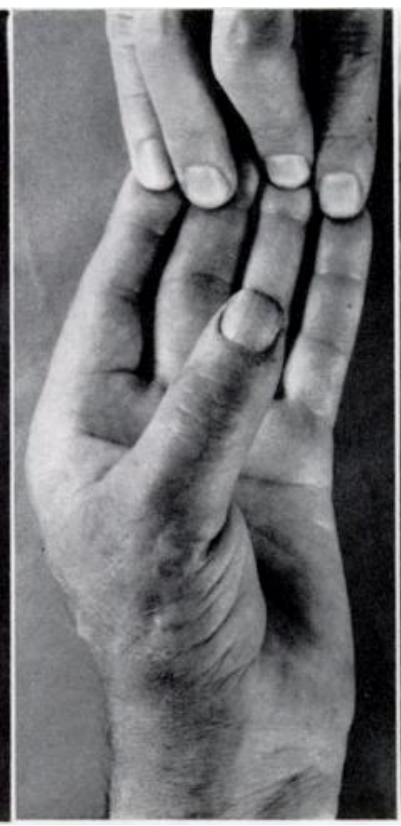

FIG. 4

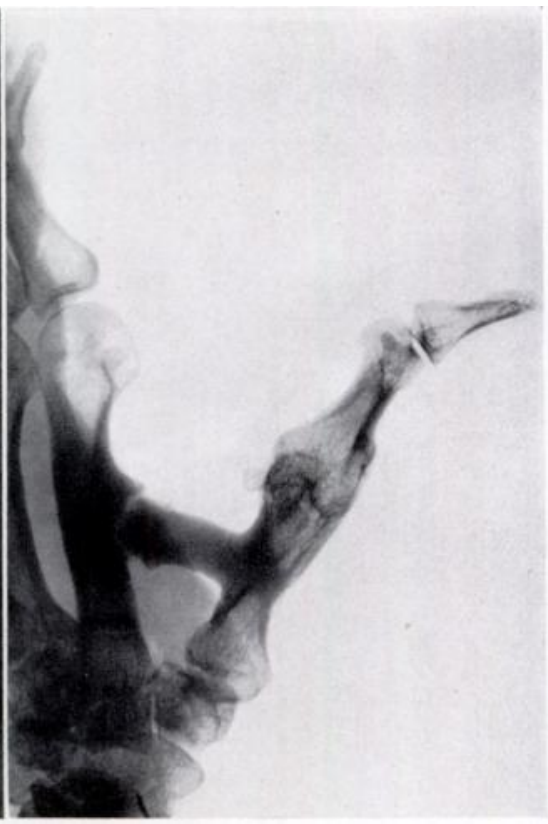

C

Case M84. Pseudarthrosis at the second metacarpal end of the graft, but a useful range of active opposition. Note the secondary rotation arthrodesis of the metacarpo-phalangeal joint of the thumb.

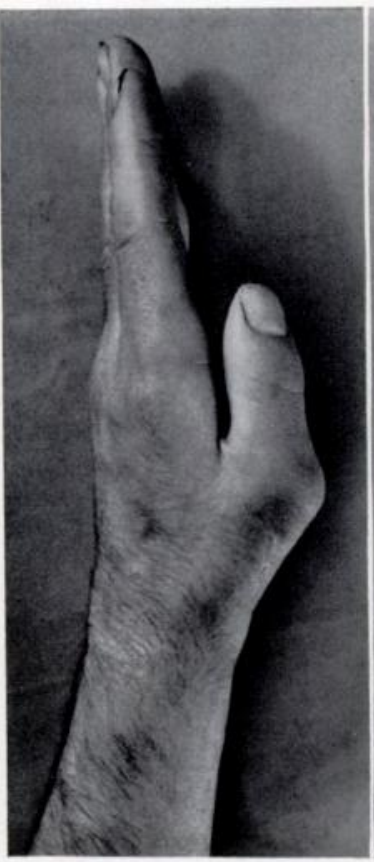

A

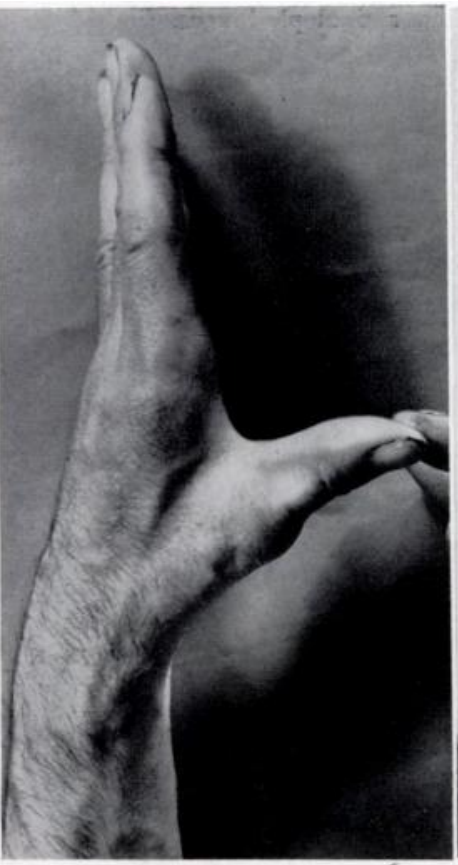

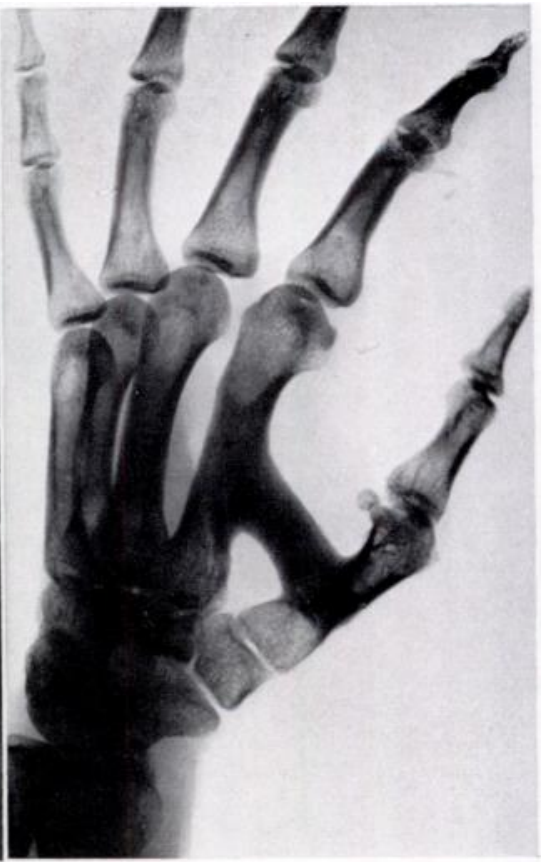

FIG. 5

Case S104. To show the acquired deformity of "pollex valgus" following an opponens bone graft. Note the incomplete rotation of the first metacarpal (A) and the degree of passive correction allowed (B). In the relaxed position (A) the tendon of extensor longus pollicis is displaced to the ulnar side like the tendon of extensor longus hallucis in hallux valgus. 
1) Failure of fusion-Five grafts failed to fuse at one or other end but never at both. In two of these cases absorption of the graft allowed the adduction deformity to recur and there was evidence to suggest that inadequate fixation after operation was the cause. Where there was failure of fusion to the second metacarpal the disability was surprisingly slight ; by using the long extensor and flexor tendons of the thumb alternately, the patient gained a range of movement through an arc the centre of which was the pseudarthrosis (Fig. 4). Foerster had a similar experience and regarded pseudarthrosis at one end as a positive advantage. 2) Sepsis-Sepsis occurred in three cases where the skin incision overlaid the graft, but with curved incisions this complication has not been seen. In one case a sequestrum consisting of most of the graft was removed, but in spite of this a firm bony strut was formed (Fig. 3C).

3) Failure to achiere full opposition-In five cases, although the thumb became fixed in full palmar abduction, rotation of the first metacarpal into full opposition was not maintained;

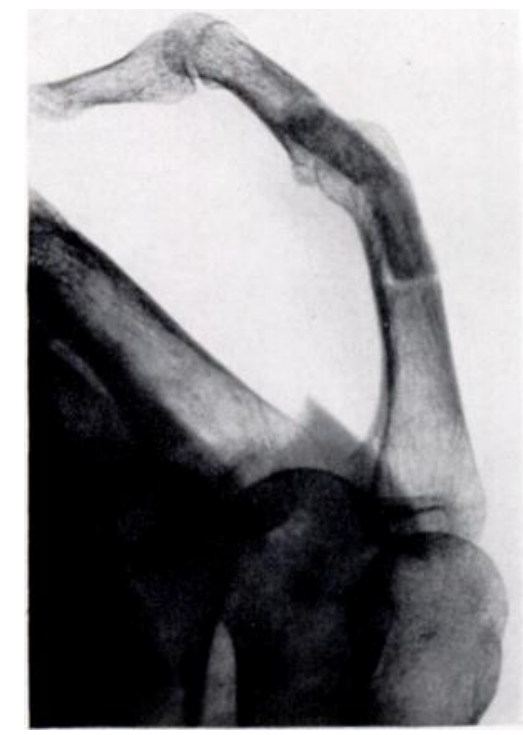

FIG. 6

Case (;41. In addition to the thenar paralysis there was complete intrinsic paralysis of the index finger; arthrodesis of the proximal interphalangeal joint has been performed.

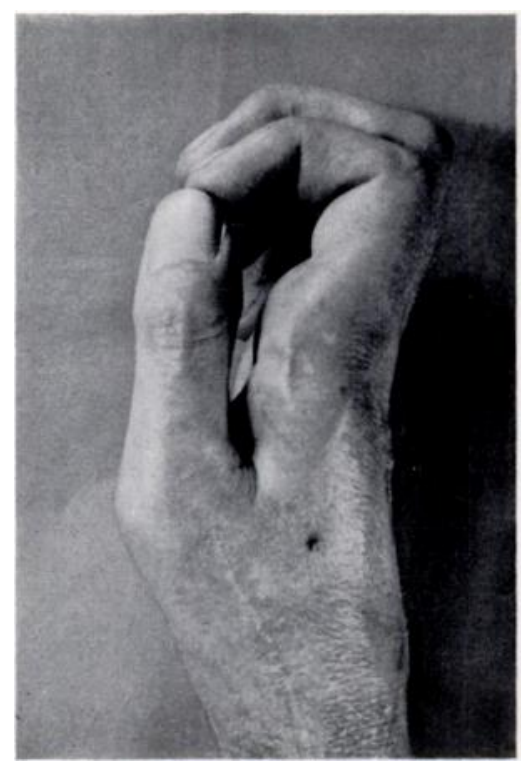

FIG. 7

Case 1263. Another case showing incomplete rotation of the first metacarpal

thus the pad of the thumb met the side of the distal phalanx of the index and gave a weak grip. In one case arthrodesis of the metacarpo-phalangeal joint of the thumb in slight flexion and correct rotation was subsequently performed (Fig. 4C). In another case a fracture of the first metacarpal occurred at the site of inlay during operation, and the opportunity to perform a rotation osteotomy was accepted with success.

4) Combined paralysis of the first dorsal interosseous and lumbrical muscles-With a thenar paralysis, although the thumb lies in the plane of the palm, there is a strong grip between the pad of the thumb and the side of the second metacarpo-phalangeal joint. In such a case, if the thumb is maintained in full abduction without full rotation the grip is transferred distally so that the side of the thumb and the side of the distal phalanx of the index meet. Usually this is not a great disability; but if the first dorsal interosseous and lumbrical are 
D. M. BROOKS

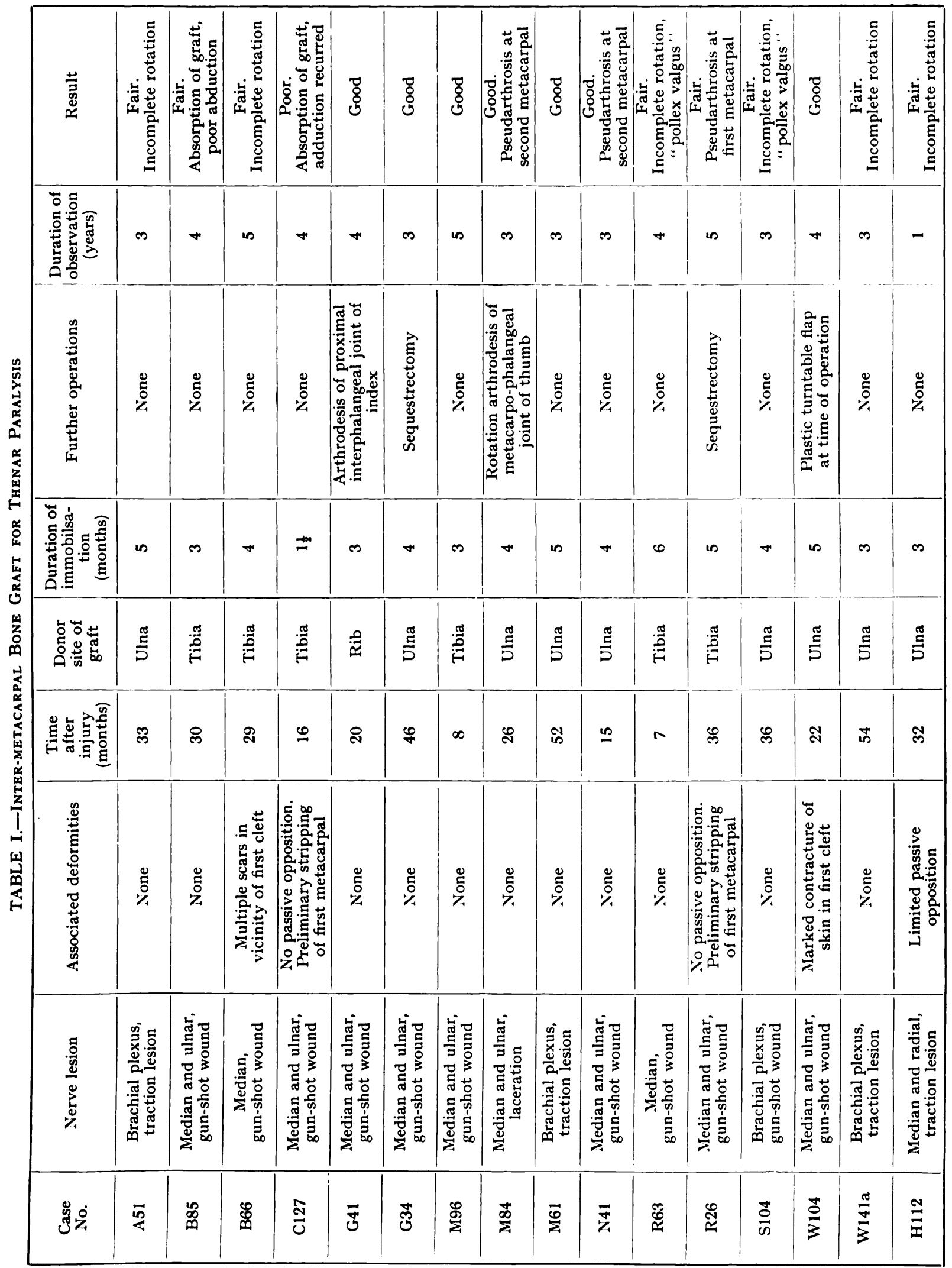

THE JOURNAL OF BONE AND JOINT SURGERY 
paralysed the grip is weakened considerably since radial stabilisation of the index finger is impossible. Furthermore, if full abduction and opposition are achieved by means of a bone graft and there is simultaneous paralysis of the first dorsal interosseous and lumbrical muscles, the tip of the index may fail to reach the pad of the thumb, because the long flexors, acting alone, cause the finger to fold up. This disability was overcome in one case by performing arthrodesis of the proximal interphalangeal joint of the index in about 35 degrees of flexion (Fig. 6).

5) "Pollex valgus"-This acquired deformity has been seen twice (Fig. 5). It may have been due to paralysis of abductor pollicis brevis; on the other hand both these cases showed incomplete rotation of the first metacarpal (Fig. 7) with ulnar displacement of the tendon of extensor pollicis longus; and it seems more probable that this is the important etiological factor. The disability from such a deformity is more apparent than real because the abducted position of the first metacarpal permits a useful grasp even though opposition is defective.

\section{SUMMARY AND GONGLUSIONS}

1. Sixteen cases of thenar paralysis are reviewed in which a bone graft was inserted between the first and second metacarpals to maintain fixed abduction and opposition of the thumb.

2. The technique of the operation is described and the causes of failure are discussed.

3. The operation is intended for those cases in which tendon transplantation to restore active opposition of the thumb is unsuitable. Rotation of the first metacarpal into full opposition is the most important feature of the operation.

I am most grateful to $\mathrm{Mr} \mathrm{H}$. J. Seddon for his helpful criticism; to the staff of the Photographic Department of the Institute for the clinical photographs; and to Miss Diana Stanley for the drawings.

\section{REFERENCES}

Bunnell, S. (1948): Surgery of the Hand. Second edition. Philadelphia: J. B. Lippincott Company. Foerster, O. (1930): Acta Chirurgica Scandinavica, 67, 351.

Highet, W. B. (1943): Lancet, 1, 227.

IRWIN, C. E. (1942): Southern Medical Journal, 35, 257.

LyLe, H. H. M. (1926): Annals of Surgery, 84, 288.

MüLlER, G. M. (1949): Journal of Bone and Joint Surgery, $31-B, 540$

Rowntree, T. (1949): Journal of Bone and Joint Surgery, 31-B, 505.

Stiles, H. J., and Forrester-Brown, M. F. (1922): Treatment of Injuries of the Peripheral Spinal Nerves. Oxford Medical Publications. London: Henry Frowde and Hodder \& Stoughton.

Thомpson, C. F. (1942): Journal of Bone and Joint Surgery, 24, 907.

Vol. $31 \mathrm{~B}$, No. 4, NOVEMBER 1949 